\title{
A High-performance Planar-type UV Photodetector Based on High-quality $\mathrm{CH}_{3} \mathrm{NH}_{3} \mathrm{PbCl}_{3}$ Perovskite Single Crystals
}

Zhen Cheng,,$^{\dagger+}$ Kewei Liu, $,^{\dagger},,^{*}$ Jialin Yang, ${ }^{\dagger+}$ Xing Chen, ${ }^{\dagger}$ Xiuhua Xie, ${ }^{\dagger}$ Binghui Li, ${ }^{+}$ Zhenzhong Zhang, ${ }^{+}, \dot{+}$ Lei Liu, ${ }^{\dagger}, \dot{+}$ Chongxin Shan, ${ }^{\S}$ Dezhen Shen ${ }^{+,+\dot{*}, *}$

$\uparrow$ State Key Laboratory of Luminescence and Applications, Changchun Institute of Optics, Fine Mechanics and Physics, Chinese Academy of Sciences, Changchun 130033, P.R. China

\$ Center of Materials Science and Optoelectronics Engineering, University of Chinese Academy of Sciences, Beijing 100049, P.R. China

$\S$ School of Physics and Engineering, Zhengzhou University, Zhengzhou 450052, P.R. China

*Corresponding authors, email: *liukw@ciomp.ac.cn (K.L.), shendz@ciomp.ac.cn (D.S.). 


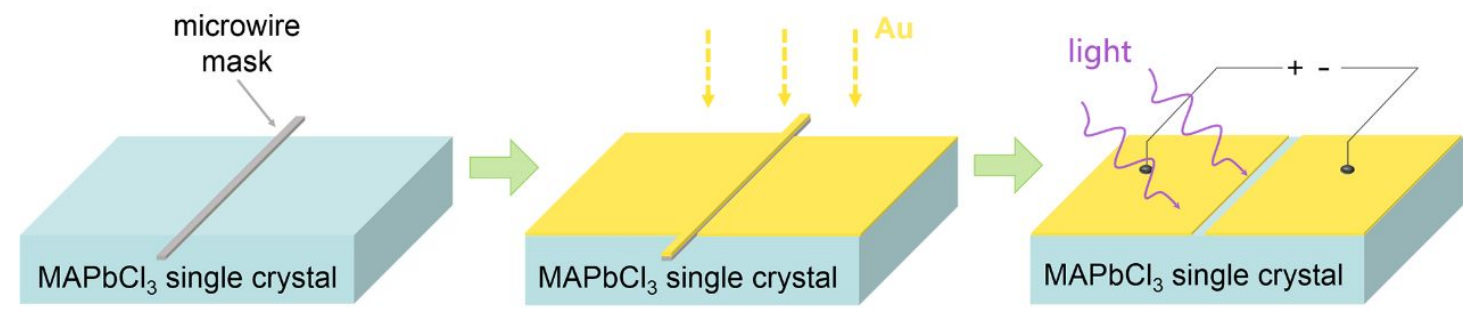

Figure S1. Schematic of the fabrication process of photodetectors based on $\mathrm{MAPbCl}_{3}$ single crystals.

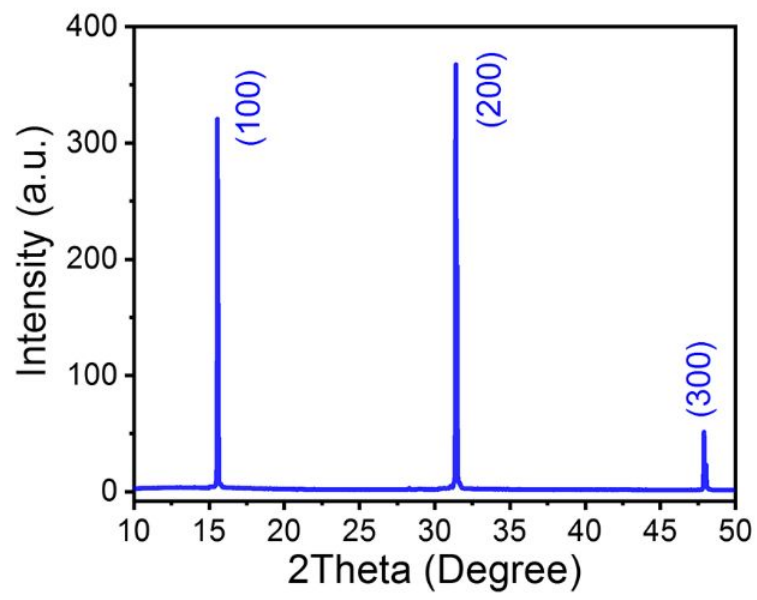

Figure S2. High-resolution XRD $2 \theta$ scan on the small faces of the $\mathrm{MAPbCl}_{3}$ crystal. 
Table S1. Comparison of the property parameters of $\mathrm{MAPbCl}_{3}$ single crystals obtained by two-step $\left(45 \& 60^{\circ} \mathrm{C}\right)$ and one-step $\left(60^{\circ} \mathrm{C}\right)$ methods.

\begin{tabular}{cll}
\hline Sample & Trap density & Hole mobility \\
\hline 1 (two-step) & $7.9 \times 10^{9} \mathrm{~cm}^{-3}$ & $\sim 64 \mathrm{~cm}^{2} \mathrm{~V}^{-1} \mathrm{~s}^{-1}$ \\
2 (two-step) & $8.2 \times 10^{9} \mathrm{~cm}^{-3}$ & $\sim 58 \mathrm{~cm}^{2} \mathrm{~V}^{-1} \mathrm{~s}^{-1}$ \\
3 (one-step) & $6.6 \times 10^{10} \mathrm{~cm}^{-3}$ & $\sim 34 \mathrm{~cm}^{2} \mathrm{~V}^{-1} \mathrm{~s}^{-1}$ \\
4 (one-step) & $4.3 \times 10^{10} \mathrm{~cm}^{-3}$ & $\sim 41 \mathrm{~cm}^{2} \mathrm{~V}^{-1} \mathrm{~s}^{-1}$ \\
\hline
\end{tabular}

Table S2. Comparison of the key performance parameter of photodetectors based on $\mathrm{MAPbCl}_{3}$ single crystals obtained by two-step $\left(45 \& 60{ }^{\circ} \mathrm{C}\right)$ and one-step $\left(60{ }^{\circ} \mathrm{C}\right)$ methods.

\begin{tabular}{ccccc}
\hline Device & Dark current & Responsivity & Rise time & Decay time \\
\hline 1 (two-step) & $7.36 \times 10^{-8} \mathrm{~A}$ & $3.73 \mathrm{~A} \mathrm{~W}^{-1}$ & $130 \mathrm{~ns}$ & $368 \mu \mathrm{s}$ \\
2 (two-step) & $8.31 \times 10^{-8} \mathrm{~A}$ & $2.56 \mathrm{~A} \mathrm{~W}^{-1}$ & $481 \mathrm{~ns}$ & $375 \mu \mathrm{s}$ \\
3 (one-step) & $9.7 \times 10^{-8} \mathrm{~A}$ & $0.86 \mathrm{~A} \mathrm{~W}^{-1}$ & $0.06 \mathrm{~s}$ & $0.72 \mathrm{~s}$ \\
4 (one-step) & $2.32 \times 10^{-7} \mathrm{~A}$ & $0.294 \mathrm{~A} \mathrm{~W}^{-1}$ & $0.12 \mathrm{~s}$ & $2.82 \mathrm{~s}$ \\
\hline
\end{tabular}

